\title{
Politeness and formulaicity Evidence from Cypriot Greek
}

\author{
Marina Terkourafi \\ University of Cambridge
}

In Brown and Levinson's politeness theory, the degree of indirectness of an utterance is presumed to be commensurate with the sum of the variables of Distance, Power and Ranking of an imposition. This claim was tested with reference to a corpus of spontaneous requests from Cypriot Greek. It emerged that, rather than motivating the choice of a 'generic' politeness strategy corresponding to a certain degree of indirectness, particular combinations of values of extra-linguistic variables (such as sex, age and social class of interlocutors, relationship between them, setting) tend to co-occur with particular combinations of the values of linguistic variables (type of verb, subjective modality, number/person). It is proposed that the latter combinations constitute formulae accessed during the production/interpretation of polite discourse in a holistic manner alongside perceivable features of the extra-linguistic context.

Keywords: politeness, formulaicity, conventional knowledge, frames, Cypriot Greek

\section{Background and objectives}

Brown and Levinson's politeness theory (1978/1987; henceforth B\&L) is based on two important assumptions: that interlocutors are rational, and that they are endowed with face (B\&L: 61). In their theory, every speech act is potentially face-threatening to an aspect of the hearer's or the speaker's face. Although face-threat is imminently present, it is so in varying degrees, represented as the seriousness or weightiness $(\mathrm{W})$ of a face-threatening act (FTA $x$ ). Interlocutors calculate this using the formula $\mathrm{W}_{x}=\mathrm{D}(\mathrm{S}, \mathrm{H})+\mathrm{P}(\mathrm{H}, \mathrm{S})+\mathrm{R}_{x}$, where $\mathrm{D}(\mathrm{S}, \mathrm{H})$ is the social distance between the speaker and the hearer, $\mathrm{P}(\mathrm{H}, \mathrm{S})$ is the power of the 
hearer over the speaker, and $\mathrm{R}_{x}$ is the ranking of the imposition that FTAx entails in the culture in question (B\&L: 76). As the weightiness of an FTA increases, interlocutors move upwards along a scale of increasing indirectness represented as a hierarchy of strategies (Figure 1). These five strategies, as well as their relative ordering, are claimed to be universal. However, the weightiness of a particular FTA $x$, as computed based on the values of $\mathrm{D}, \mathrm{P}$ and $\mathrm{R}_{x}$ pertaining to this act, will be culture-specific.

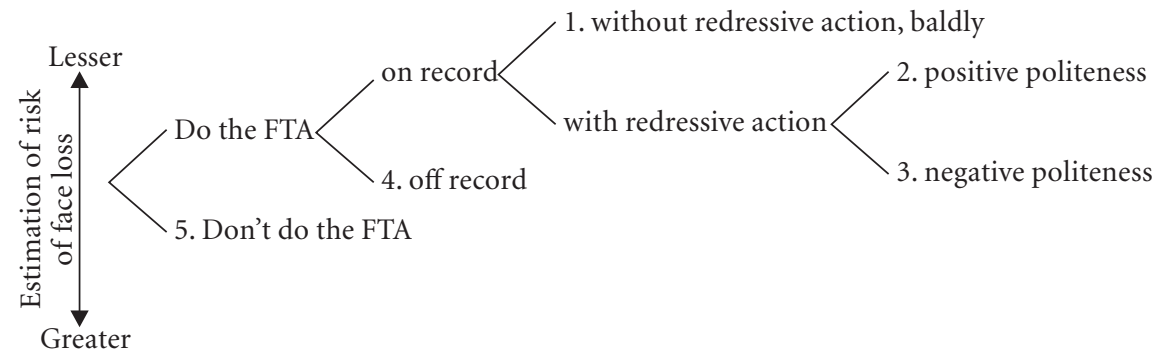

Figure 1. Strategies for performing FTAs (B\&L: 60)

This paper aims to test Brown and Levinson's claim that the degree of indirectness of an utterance realising an FTA $x$ is commensurate with the sum of the values that the speaker assigns to $\mathrm{D}, \mathrm{P}$ and $\mathrm{R}_{x}$ drawing on a corpus of spontaneous conversational data from Cypriot Greek (henceforth CG).

The discussion of realisations of requests in CG reveals that speakers regularly have recourse to a small number of 'formulae', where a formula is defined as a fully specified morpho-phonological surface realisation of a VP whose head is subject to lexical and/or semantic constraints. Politeness formulae thus defined fall under what Pawley and Syder (1983:208ff.) term 'lexicalised sentence stems'. One distinctive property of lexicalised sequences is that they are 'social institutions' (ibid.: 209): they are, to some extent, arbitrarily chosen among synonymous expressions, as the standard way of expressing a particular culturally authorised meaning (ibid.: 211).

Two observations currently warrant use of the term 'formulae' to refer to preferred realisations of requests in the data. First, given a particular combination of extra-linguistic features, speakers' choices are not equally distributed among potential realisations of a politeness strategy ${ }^{1}$ - as one would expect if $\mathrm{W}$ values guided merely the selection of a generic politeness strategy - but cluster around a particular, lexically-specific realisation, sometimes specified down to the level of phonetic detail. Second, different realisations of the same 
politeness strategy are preferred in different contexts (i.e. combinations of extra-linguistic features). ${ }^{2}$ In other words, when a particular combination of extra-linguistic features is encountered, speakers appear to opt for concrete, fully specified, linguistic realisations rather than a general level of indirectness, or politeness strategy. This intimate relationship between lexically-specific realisations of politeness strategies (i.e. combinations of linguistic features) and contexts of use (i.e. combinations of extra-linguistic features) cannot be captured within Brown and Levinson's scheme, where particular realisations of strategies and particular combinations of extra-linguistic features are abstracted away from, a correlation being drawn instead between politeness strategies and $\mathrm{W}$ values. It would seem that, while Brown and Levinson's universalising scheme may be applicable at an abstract level, describing the regularities observed in the CG data necessitates taking a further step. The potential of a frame-based approach to politeness is explored in this light. Such an approach relies on a notion of cognitive frames, defined as experientially-based structures of expectations, and is exemplified in what follows by proposing some frames for requests in CG.

\section{Data collection and analysis}

This study is based on 1,486 spontaneous realisations of requests in CG originating in recordings of adult native CG speakers conducted in the four major urban centres of the island, and in a variety of settings. Appealing to a general dimension of desirability of an act predicated of the speaker or of the addressee in an utterance of a sentence (Sperber \& Wilson 1986/1995:250-1), requests are generically defined as acts desirable to the speaker. In addition to avoiding theoretical objections as to the adequacy of proposed classifications of speech acts, this methodological decision is supported by empirical evidence suggesting that speech-act theoretical descriptions play no actual role in comprehension (Good MS, reported in Geis 1995:31). The desirability of an act can be inferred based on the propositional content of the utterance and features of the situational context, including the addressee's uptake, even if the illocutionary force of the utterance cannot be further clarified. Thus identified and subsequently semi-phonologically transcribed, realisations of requests were analysed for a number of variables, linguistic and extra-linguistic.

Among the linguistic devices available in Modern Greek for performing requests (Sifianou 1992:125ff.), the present analysis takes into account: the 
presence/absence of a main-clause verb predicating an act $A$ of the speaker or of the addressee, the type of main-clause verb, the subjective modality expressed by it, and the combination of number/person for which it is marked. ${ }^{3}$ In principle, each of these may be related to one or more strategies from Brown and Levinson's hierarchy (Figure 1). However, such a move is justifiable only on the assumption that combinations of these devices are in some way equivalent (by, e.g., falling under the same strategy), and can therefore be used interchangeably. ${ }^{4}$ Aiming precisely to test the validity of this assumption, this study refrains from taking this step.

Extra-linguistic features of the situation considered during the analysis of the data include the interlocutors' sex, age, and social class, the relationship between them, the setting of the exchange, and whether the speech act performed occurs for the first time or is repeated. These provide the input for assessing the situation-specific values of $\mathrm{D}, \mathrm{P}$ and $\mathrm{R}_{x}$ in the data collected. ${ }^{5}$ This move is based on the requirement for a consistent way of assessing the values of these sociological variables within a community, i.e. one which is not (wholly) idiosyncratic. For, if this requirement is relaxed, not only does the theory become unfalsifiable, it also becomes hard to explain how interlocutors may agree on their assessment of the FTA-specific values of $\mathrm{D}, \mathrm{P}$ and $\mathrm{R}_{x}$ (hence perceive each other to be polite), if not wholly by coincidence.

To establish whether, when particular values of $\mathrm{D}, \mathrm{P}$ and $\mathrm{R}_{x}$ are operative, speakers opt for a general level of indirectness realised as various combinations of values of the linguistic variables considered all falling under the same strategy from Brown and Levinson's proposed hierarchy (Figure 1), or a more direct correlation holds between particular combinations of values of the extralinguistic and linguistic variables, frequencies of co-occurrence of combinations of values of the extra-linguistic variables with combinations of values of the linguistic variables were investigated.

\section{Overview of the findings}

In the analysis which follows, speakers' preferences for particular combinations of type of verb, subjective modality, and number/person are first located in the data collected, and the difficulties of accounting for them within Brown and Levinson's scheme are discussed. To overcome such difficulties, sociolinguistically informed insights are appealed to. Each subsection closes by proposing a suitable frame, which captures the conventional relationship between the formulae discussed and their contexts of use. 
3.1 exo-indicative with rising intonation-2nd plural/3rd singular

In Brown and Levinson's hierarchy of strategies, exo, 'I-have'-indicative with rising intonation-2PL (realisation: /eçete?/) and exo-indicative with rising intonation-3sg (realisation: /eçi?/) both fall under negative politeness: they realise sub-strategy 1, 'be conventionally indirect', by seeking to confirm whether the preparatory condition regarding the existence of goods requested holds (B\&L: 137), and sub-strategy 7, 'impersonalise S and H’ (B\&L: 190ff.)

(1) [Speaker: male, 31-50, middle-class; Addressee: female, 18-30, workingclass; Setting: at work (pub); Relationship: new customer to salesperson] eçeter $\mathrm{p}^{\mathrm{h}}$ inats?

have-IND-2PL, peanuts?

'Do you-2PL have some peanuts?'

(2) [Speaker: female, 31-50, working-class; Addressee: male, 31-50, working-class; Setting: at work (open-air market); Relationship: new customer to salesperson]

eçi mikres pu na min exun sçeðia pano? aspro have-IND-3SG small \{socks\} that SP not have-IND-3PL patterns on? white 'Are there any small plain ones? In white.'

However, the two expressions are differentially used in the data collected. While both are used by new customers making first requests of salespersons, /eçete?/is preferred by middle-class speakers (Table 1 ) and is almost absent from the speech of working-class speakers, where it is replaced by /eçi?/ (Table 2). ${ }^{6}$ Similarly, working-class salespersons receive more /eçi?/ (Table 3) compared to middle-class salespersons (Table 4). 
Table 1. Frequencies of occurrence of combinations of type of verb-subjective modality-number/person used by middle-class customers requesting something for the first time of a salesperson with whom they are not familiar

\begin{tabular}{|c|c|}
\hline leçete?/ ('do you-PL have?') & 5 \\
\hline / $\theta$ elo/ ('I want') & 4 \\
\hline /eçi?/ ('is/are there?') & 4 \\
\hline ACTION VERB-IMPERATIVE-2nd SINGULAR & 2 \\
\hline ACTION VERB-SUBJUNCTIVE-1st SINGULAR & 1 \\
\hline ACTION VERB-INDICATIVE+RISING INTONATION-1st PLURAL & 1 \\
\hline ACTION VERB-FUTURE-1st PLURAL & 1 \\
\hline ACTION VERB-FUTURE+RISING INTONATION-1st SINGULAR & 1 \\
\hline SPEECH ACT VERB-IMPERATIVE-2nd SINGULAR & 1 \\
\hline SPEECH ACT VERB-IMPERATIVE-2nd PLURAL & 1 \\
\hline /Oelume/ ('we want') & 1 \\
\hline 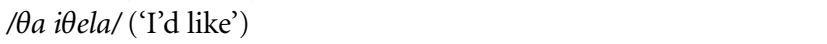 & 1 \\
\hline /exume?/ ('do we have?’) & 1 \\
\hline /eçis?/ ('do you-sg have?’) & 1 \\
\hline /boro?/ ('can I?') & 1 \\
\hline /ðen eçi?/ ('isn’t/aren’t there?’) & 1 \\
\hline Indirect statement using a main-clause verb other than the above & 5 \\
\hline Total & 32 \\
\hline
\end{tabular}

Table 2. Frequencies of occurrence of combinations of type of verb-subjective modality-number/person used by working-class customers requesting something for the first time of a salesperson with whom they are not familiar

\begin{tabular}{|c|c|}
\hline ACTION VERB-IMPERATIVE-2nd SINGULAR & 5 \\
\hline /eçi?/ ('is/are there?') & 3 \\
\hline ACTION VERB-SUBJUNCTIVE+RISING INTONATION-2nd PLURAL & 2 \\
\hline /eçis?/ ('do you-sg have?’) & 2 \\
\hline ACTION VERB-SUBJUNCTIVE-1st SINGULAR & 1 \\
\hline ACTION VERB-SUBJUNCTIVE-1st PLURAL & 1 \\
\hline ACTION VERB-SUBJUNCTIVE-2nd SINGULAR & 1 \\
\hline ACTION VERB-SUBJUNCTIVE+RISING INTONATION-2nd SINGULAR & 1 \\
\hline /日eli/ ('he/she wants') & 1 \\
\hline /Aelo?/ ('I want?’) & 1 \\
\hline /ïela// ('I wanted') & 1 \\
\hline /Oelame?// ('we wanted?') & 1 \\
\hline /boro?// ('can I?') & 1 \\
\hline /eçete?/ ('do you-PL have?') & 1 \\
\hline Indirect statement using a main-clause verb other than the above & 3 \\
\hline Indirect statement not using a main-clause verb & 1 \\
\hline Total & 26 \\
\hline
\end{tabular}


Table 3. Frequencies of occurrence of combinations of type of verb-subjective modality-number/person received by working-class salespersons from customers with whom they are not familiar and who are requesting something for the first time

\begin{tabular}{|c|c|}
\hline /eçi?/ ('is/are there?’) & 4 \\
\hline ACTION VERB-IMPERATIVE-2nd SINGULAR & 4 \\
\hline /eçete?/ ('do you-PL have?’) & 3 \\
\hline /eçis?/ ('do you-sg have?’) & 2 \\
\hline /Oelo/ ('I want') & 2 \\
\hline /ðen eçi?/ ('isn’t/aren’t there?’) & 1 \\
\hline ACTION VERB-SUBJUNCTIVE-1st SINGULAR & 1 \\
\hline ACTION VERB-SUBJUNCTIVE-2nd SINGULAR & 1 \\
\hline ACTION VERB-SUBJUNCTIVE-1st PLURAL & 1 \\
\hline /Oelume/ ('we want') & 1 \\
\hline / $\theta e l i /$ ('he/she wants') & 1 \\
\hline 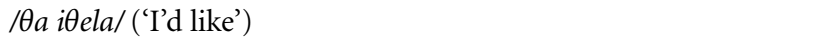 & 1 \\
\hline /iもela/ ('I wanted') & 1 \\
\hline /boro?/('can I') & 1 \\
\hline Indirect statement using a main-clause verb other than the above & 2 \\
\hline Total & 26 \\
\hline
\end{tabular}

Table 4. Frequencies of occurrence of combinations of type of verb-subjective modality-number/person received by middle-class salespersons from customers with whom they are not familiar and who are requesting something for the first time

\begin{tabular}{|c|c|}
\hline /eçete?/ ('do you-PL have?’) & 3 \\
\hline ACTION VERB-IMPERATIVE-2nd SINGULAR & 3 \\
\hline /eçi?/ ('is/are there?') & 2 \\
\hline ACTION VERB-SUBJUNCTIVE+RISING INTONATION-2nd PLURAL & 2 \\
\hline$/ \theta e l o /(' I$ want') & 2 \\
\hline /boro?/('can I') & 2 \\
\hline ACTION VERB-SUBJUNCTIVE-1st SINGULAR & 1 \\
\hline ACTION VERB-INDICATIVE+RISING INTONATION-1st PLURAL & 1 \\
\hline ACTION VERB-SUBJUNCTIVE+RISING INTONATION-2nd SINGULAR & 1 \\
\hline ACTION VERB-FUTURE-1st PLURAL & 1 \\
\hline ACTION VERB-FUTURE+RISING INTONATION-1st SINGULAR & 1 \\
\hline SPEECH ACT VERB-IMPERATIVE-2nd SINGULAR & 1 \\
\hline SPEECH ACT VERB-IMPERATIVE-2nd PLURAL & 1 \\
\hline /日elo?/ ('I want?') & 1 \\
\hline /Oelame?/ ('we want') & 1 \\
\hline /exume?/ ('do we have?’) & 1 \\
\hline /eçis?/ ('do you-sg have?’) & 1 \\
\hline Indirect statement using a main-clause verb other than the above & 6 \\
\hline Indirect statement not using a main-clause verb & 1 \\
\hline Total & 32 \\
\hline
\end{tabular}


These findings appear perplexing if the two expressions are 'equivalently' indirect (in the sense of both falling under negative politeness, and realising the same sub-strategies of this strategy). Middle- and working-class speakers' consistently different solutions to the task of impersonalising the addressee suggest that, when requesting something for the first time from a salesperson they are not familiar with, speakers are also performing an act of identity. Le Page and Tabouret-Keller (1985:182) define this as follows:

"We can only behave according to the behavioural patterns of groups we find it desirable to identify with to the extent that:

i. we can identify the groups

ii. we have both adequate access to the groups and ability to analyse their behavioural patterns

iii. the motivation to join the groups is sufficiently powerful, and is either reinforced or reversed by feedback from the groups

iv. we have the ability to modify our behaviour."

To the extent that non-literal use of the 2nd person plural in CG marks instances of standardising speech (Terkourafi 2001a), it is constrained by both having access to the standard code, and the extent to which the speaker finds identifying him/herself with 'the group of standard speakers' desirable in the situation at hand. Middle-class speakers have greater access to Standard Modern Greek (henceforth SMG) than working-class speakers, in that they have more opportunities to interact with Mainland Greeks, either in Greece, where they travel regularly to study, on holiday, or on business trips, or in Cyprus, where Mainland Greeks often hold short-term contracts in white-collar jobs. Being socially stratified as a result, non-literal use of the 2 nd person plural constitutes an outward manifestation of middle-class identity, to be called upon when asserting this identity is judged desirable. The financial implications of a commercial exchange make requesting something for the first time from a salesperson with whom one is not familiar just such an occasion, when asserting one's middle-class identity can have desirable consequences for the speaker. However, this motivation is constrained by the desire to accommodate one's speech to that of one's addressee. Consequently, middle-class speakers tend to use /eçete?/ more with middle-class addressees than with working-class ones.

/eçete?/ and /eçi?/ thus emerge as representing different norms, to which speakers tend or which they avoid depending (a) on the degree to which they have access to them, and (b) on their desire to present themselves in a certain way, which subsumes the wish to accommodate their speech to that of their addressees. This analysis finds support in the phonetic realisations of the two 
variants, which largely conform to the rules of SMG and CG respectively: /eçete?/ is realised as eçete, i.e. with suspension of a Cypriot-specific rule of softening of $/ \mathrm{x} /$ to [ $\left.\int\right]$ (Newton 1972:51), five out of six times (83.34\%), while /eçi?/ is realised as efi, i.e. applying softening of / $\mathrm{x} /$ to [f], six out of eight times (75\%). The discovery of consistency in speakers' choices down to the level of phonetic detail argues strongly in favour of the formulaic character of these expressions. Rather than creating them anew based on nonce calculations of D, $\mathrm{P}$ and $\mathrm{R}_{x}$, speakers appear to retrieve them in a holistic manner when encountering particular combinations of extra-linguistic features. The extra-linguistic factors jointly determining use of these expressions in the data collected are the relationship between interlocutors, the order of appearance of the speech act, the speaker's social class, and to a lesser extent his/her sex and age relative to the addressee, as well as the addressee's social class (Frames 1,2). ${ }^{7}$

Frame 1. Proposed frame for /eçete?/ as the preferred formula for requests

Sex of speaker: female, male

Sex of addressee: any

Relative age of interlocutors: younger to older, older to younger, same

Social class of speaker: middle

Speech act: request

Occurs for the: 1st time
Social class of addressee: middle, working

Relationship: new customer to salesperson

Setting: at work

leçete?/

Frame 2. Proposed frame for /eçi?/ as the preferred formula for requests

Sex of speaker: male, female

Sex of addressee: male, female

Relative age of speaker and addressee: same, any

Social class of speaker: working

Speech act: request

Occurs for the: 1st time
Social class of addressee: working, middle

Relationship: new customer to salesperson

Setting: at work

\section{leçi?/}

\subsection{Aelo-conditional-1st singular}

The polite formulae discussed so far concern the realisation of requests at work. In requests performed during formal discussions or radio/TV interviews, most preferred are two further combinations, $\theta$ elo, 'I-want'-indicative-1SG (realis-ation: $/ \theta e l o /)$ and $\theta e l o-c o n d i t i o n a l-1 S g$ (realisation: / $\theta$ a i $\theta$ ela/) (Tables 5-8). 
(3) [Speaker: male, 31-50, middle-class; Addressee: female, 31-50, middle-

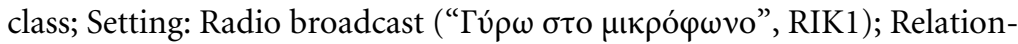
ship: interviewer to interviewee]

$\theta$ elo na apandisumen is ton akroatin pu ton afisamen (.) eh want-IND-1SG SP answer-DEP-1PL to the-ACC listener-ACC that him-ACC leave-PAST-PERF-1PL(.) er

'I'd like $\{$ us $\}$ to answer the listener that we left (.) er'

(4) [Speaker: female, 31-50, middle-class; Addressee: male, 31-50, middle-

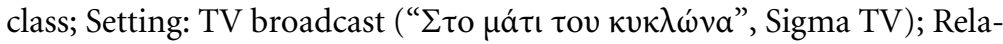
tionship: interviewee to interviewer]

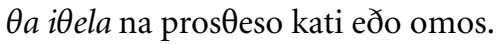

FP want-PAST-IMPERF-1SG SP add-DEP-1SG something here though.

'I would like to add something here though.' 
Table 5. Frequencies of occurrence of combinations of type of verb-subjective modality-number/person used by interviewers in formal settings

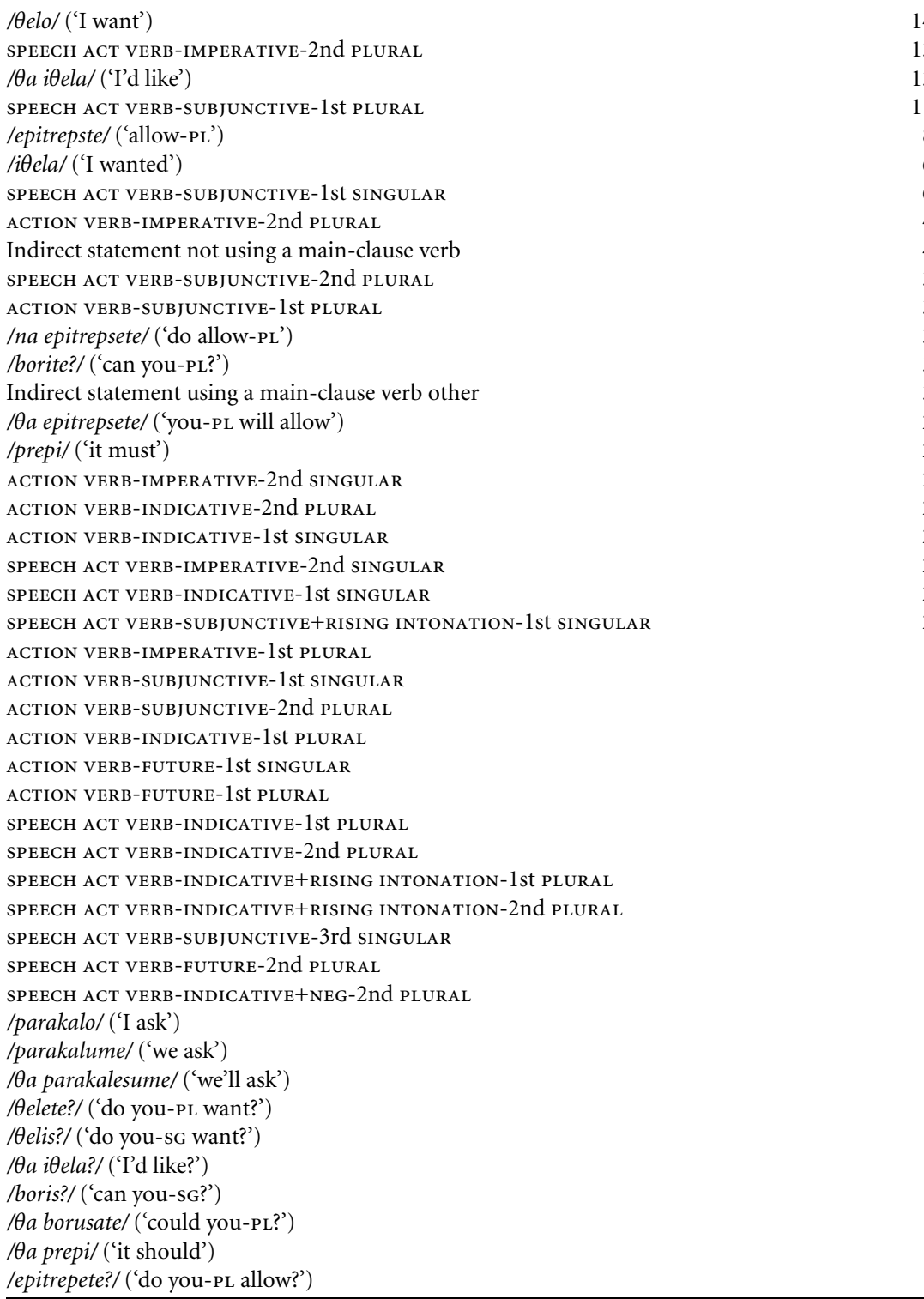


Table 6. Frequencies of occurrence of combinations of type of verb-subjective modality-number/person used by men

$\begin{array}{lc}/ \theta e l o /(' I \text { want') } & 21 \\ / \theta \text { i i } \theta \text { ela/ ('I'd like') } & 18 \\ \text { SPEECH ACT VERB-IMPERATIVE-2nd PLURAL } & 16 \\ \text { SPEECH ACT VERB-SUBJUNCTIVE-1st SINGULAR } & 16 \\ \text { SPEECH ACT VERB-SUBJUNCTIVE-1st PLURAL } & 13 \\ \text { /epitrepste/('allow-PL') } & 10\end{array}$

No main-clause verb used 7

ACTION VERB-IMPERATIVE-2Nd SINGULAR 6

ACTION VERB-IMPERATIVE-2nd PLURAL

/prepi/ ('it must') 6

SPEECH ACT VERB-SUBJUNCTIVE-2nd PLURAL

ACTION VERB-SUBJUNCTIVE-1St PLURAL

Indirect statement using a main-clause verb other 5

SPEECH ACT VERB-INDICATIVE-1st SINGULAR 4

Ina epitrepsete/ ('do allow-PL') 3

ACTION VERB-INDICATIVE-2nd PLURAL 3

SPEECH ACT VERB-SUBJUNCTIVE+RISING INTONATION-1st SINGULAR 3

/borite?/ ('can you-PL?') 3

/borume/ ('we can') 2

/ $\theta$ a epitrepsete/ ('you-PL will allow') 2

/Oa prepi/ ('it should') 2

ACTION VERB-INDICATIVE-1st SINGULAR 2

SPEECH ACT VERB-IMPERATIVE-2nd SINGULAR 2

ACTION VERB-SUBJUNCTIVE-2nd SINGULAR 2

ACTION VERB-INDICATIVE-1st PLURAL 2

SPEECH ACT VERB-FUTURE-1st SINGULAR

ACTION VERB-IMPERATIVE-1ST PLURAL

ACTION VERB-SUBJUNCTIVE-1ST SINGULAR

ACTION VERB-PAST-1St SINGULAR 1

ACTION VERB-FUTURE-1ST SINGULAR 1

ACTION VERB-FUTURE-3rd SINGULAR 1

SPEECH ACT VERB-INDICATIVE-1ST PLURAL

SPEECH ACT VERB-INDICATIVE+RISING INTONATION+NEG-2nd PLURAL 1

SPEECH ACT VERB-PAST-1St SINGULAR 1

SPEECH ACT VERB-SUBJUNCTIVE-3rd SINGULAR 1

SPEECH ACT VERB-FUTURE+RISING INTONATION-2nd SINGULAR 1

SPEECH ACT VERB-FUTURE-2nd PLURAL

SPEECH ACT VERB-INDICATIVE+NEG-2nd PLURAL 1

/parakalo/ ('I ask') 1

/Oa parakalesume/ (we'll ask) 1

/Oelete?/('do you-PL want?')

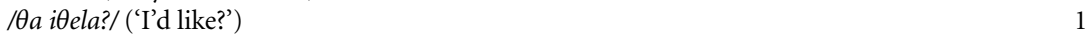

/boris?/ ('can you-SG?')

/bori/ ('it is possible') 1

lepitrepis?/ ('do you-sg allow?')

lepitrepete?/('do you-PL allow?') 1

$\begin{array}{ll}\text { Total } & 195\end{array}$ 
Table 7. Frequencies of occurrence of combinations of type of verb-subjective modality-number/person used by women in formal settings

/Oa ïela/ ('I'd like')

SPEECH ACT VERB-SUBJUNCTIVE-1st SINGULAR

ACTION VERB-IMPERATIVE-2nd SINGULAR

SPEECH ACT VERB-IMPERATIVE-2nd SINGULAR 2

SPEECH ACT VERB-IMPERATIVE-2nd PLURAL 2

ACTION VERB-SUBJUNCTIVE-1st PLURAL

$\begin{array}{ll}\text { /Oelo/ ('I want') } & 2\end{array}$

lïela/ ('I wanted') 1

ACtION VERB-SUbJUNCTIVE-2nd PLURAL 1

SPEECH ACT VERB-SUBJUNCTIVE-2nd PLURAL 1

SPEECH ACT VERB-SUBJUNCTIVE-1st PLURAL 1

SPEECH ACT VERB-SUBJUNCTIVE+RISING INTONATION-1st SINGULAR 1

SPEECH ACT VERB-INDICATIVE+RISING INTONATION-2nd PLURAL 1

SPEECH ACT VERB-INDICATIVE+RISING INTONATION-1st PLURAL 1

/parakalume/ ('we ask') 1

/ $\theta$ a borusate/ (we'll ask) 1

/prepi/ ('it must') 1

/Oa epitrepsis/ ('you-PL will allow') 1

/Oelis?/('do you-sg. want?') 1

Indirect statement using a main-clause verb other 2

No main-clause verb used $\quad 1$

Total

The first thing to note about these combinations is their attachment to the formal discussion/radio/TV setting. While / $\theta$ elo/ and $/ \theta$ a i $\theta$ ela/ constitute indirect requests by affirming the sincerity condition in the same way as /eçete?/ and /eçi?/constitute indirect requests by questioning the preparatory condition concerning the existence of goods sought (B\&L: 137), the two types of requests have clearly defined - and different - domains of application. And, whereas questioning the preparatory condition referring to the existence of goods sought may be absurd when action, rather than goods, is being requested, the same is not true of affirming the sincerity condition: affirming one's desire for some thing functions equally well as a request as questioning its availability (compare English 'Do you have NP' with 'I'd like NP'). Nevertheless, the preferences for exo-indicative with rising intonation-2PL/3SG as opposed to $\theta e l o$-indicative/ conditional-1sg. are clear. $/ \theta$ a i $\theta e l a /$ in particular is used only once in fifty-eight requests by a new customer addressing a salesperson (i.e. 1.7\%; Tables 3 and 4). In other words, although they all fall under negative politeness, these combinations of verb-subjective modality-number/person are 
Table 8. Frequencies of occurrence of combinations of type of verb-subjective modality-number/person used by interviewees in formal settings

/Oa ïela/ ('I'd like')

SPEECH ACT VERB-SUbJUNCTIVE-1st SINGULAR

/Aelo/ ('I want')

/prepi/ ('it must')

/ïela/ ('I wanted')

ACTION VERB-SUBJUNCTIVE-1st PLURAL

SPEECH ACT VERB-SUBJUNCTIVE-1st PLURAL

SPEECH ACT VERB-FUTURE-1st SINGULAR

ACTION VERB-IMPERATIVE-2nd PLURAL

SPEECH ACT VERB-IMPERATIVE-2nd PLURAL

ACTION VERB-INDICATIVE-1st PLURAL

ACTION VERB-INDICATIVE-2nd PLURAL

SPEECH ACT VERB-INDICATIVE+RISING INTONATION-1st SINGULAR

SPEECH ACT VERB-INDICATIVE+RISING INTONATION+NEG-2nd PLURAL

lepitrepste/ ('allow-PL')

/borume/(we'll ask)

Indirect statement using a main-clause verb other

No main-clause verb used

not interchangeable. Rather, they are attached to observable features of the situation - in the case of the exo-variants the type of relationship, in the case of the $\theta$ elo-variants the setting of the exchange - in a way that an account based on the sum of $\mathrm{D}, \mathrm{P}$ and $\mathrm{R}_{x}$ fails to predict.

Focusing on the two variants prevailing in requests performed for the first time during formal discussions and on radio/TV, yet more detailed predictions are possible. $/ \theta$ elo/ is favoured by interviewers addressing interviewees and by men (Tables 5,6 ), while $/ \theta$ a i $\theta$ ela/ is clearly favoured by interviewees addressing interviewers, and by women (Tables 7,8 ). Based on these findings, one may suspect that the choice between these two variants relates to the Power of the hearer over the speaker: with interviewers or men, $\mathrm{P}$ is low, and this is reflected in these speakers' choice of / $\theta$ elo/. However, interviewers and men favour $/ \theta$ elo/ only weakly. In this case, it is women's and interviewees' much more pronounced tendency to use $/ \theta$ a i $\theta$ ela/ that calls for an explanation.

To provide this, one needs to consider the relative salience of the two variants, defined as a dimension of perceptual prominence attributable to a combination of objective and subjective factors (cf. Auer et al. 1998). Whereas $/ \theta$ elo/remains the same whether in CG or SMG, / $\theta$ a i $\theta$ ela/noticeably belongs to 
the SMG code, as it contains the SMG particle of futurity $/ \theta a /$. This contrasts with the CG particle of futurity /en:a/, which is phonetically distant from its standard counterpart, the difference between the two being phonemic rather than phonetic, and lexicalised (cf. Auer et al's criteria of articulatory and perceptual distance, phonemicity, and lexicalisation). Moreover, /en:a/is written differently (when written), is areally restricted to Cyprus, and cannot co-occur with the 2nd person plural when this is used non-literally, a usage which has a distinctly standardising flavour in CG (Terkourafi 2001a; cf. Auer et al.' s criteria of representation in lay dialect writing, areal distribution, and usage in codealternation). / $\theta$ a i $\theta$ ela/thus turns out to be more salient than $/ \theta$ elo/both objectively and subjectively. According to Auer et al. (1998:163), "[d]ialect features which are perceived by the speakers as 'salient' are taken up and given up more easily and faster than those which are perceived as 'less salient"'.

Considering that $/ \theta$ a i $\theta$ ela/ is virtually exclusive to formal discussions and radio and TV settings, I would argue that this variant is not only associated with these settings, but is also a salient feature of 'media-speak' in CG. This explains its more frequent adoption compared to the less salient $/ \theta$ elo/ by speakers wishing to demonstrate their familiarity with 'media-speak' and the correspond-ing settings. Clearly, interviewees are particularly keen to demonstrate such familiarity: they do not appear on radio and TV as regularly as their hosts, but wish to present themselves as competent discussants nevertheless. Women too seek to confirm themselves as competent users of 'media-speak', as they appear on radio and TV less frequently than men. Women make up just over a quarter of interviewers recorded (5 out of 18 , or $27.8 \%$ ), and only a fifth of interviewees ( 8 out of 40 , or $20 \%$ ). By opting for $/ \theta a$ i $\theta$ ela/, women and interviewees perform an act of identity (Le Page \& Tabouret-Keller 1985; see 3.1 above): they seek to identify themselves with a particular group of speakers (in this case, the group of 'media-speakers' as represented by interviewers and by men) by selecting a salient variant characteristic of this group's speech.

The above discussion highlights two advantages of an account of polite discourse in terms of observable features of the situation summarised as frames over Brown and Levinson's principle-based account. First, as the comparison with the exo-variants shows, speakers' choice between 'equally' indirect forms (i.e. realisations of the same strategy) is not random but constrained by their differing domains of application. Within a frame-based approach, appealing to combinations of extra-linguistic features of the situation enables us to make this prediction. Second, by not abstracting away from the particular linguistic forms used, a frame-based approach allows us to assess the salience of an expression 
in addition to its indirectness, which, in conjunction with information about the relationship between interlocutors and about the sex of the speaker, enables us to predict not only which particular expressions speakers will choose, but also to what extent. Frame 3 below is the proposed frame for use of $/ \mathrm{Aa} i \mathrm{ela} / \mathrm{as}$ a polite request.

Frame 3. Proposed frame for $/ \theta$ a i $\theta$ ela/ as the preferred formula for requests

\begin{tabular}{ll} 
Sex of speaker: female, male & \multicolumn{1}{c}{ Sex of addressee: any } \\
\hline \multicolumn{1}{c}{ Relative age of speaker and addressee: same, younger to older } \\
\hline Social class of speaker: (middle) & $\begin{array}{l}\text { Social class of addressee: (middle) } \\
\text { Speech act: request }\end{array}$ \\
Relationship: interviewee to interviewer, interviewer \\
to interviewee
\end{tabular}

\section{Discussion}

The analysis presented above shows an account of speakers' preferred realisations of requests in the collected data that relies on Brown and Levinson's hierarchy of strategies and the proposed formula for computing $\mathrm{W}$ to fail on two counts. First, their hierarchy of strategies classifies linguistic expressions as more or less indirect based on the propositional content which they express, i.e. using semantically oriented criteria. ${ }^{8}$ However, uttered in a particular sociohistorical and linguistic context, linguistic expressions are at once standard or vernacular, and more or less salient. Significantly, notions of standardness, and salience, interact with indirectness (seen as a matter of inferential length) in determining speakers' choices in context.

The second reason why Brown and Levinson's approach fails to account for the CG data concerns their definitions of the three sociological variables, D, P and $\mathrm{R}_{x}$. Their approach places the emphasis on the relationship between interlocutors, i.e. on the dyad. This is clear in their association of politeness strategies with particular pay-offs (which may also take the form of assumptions) that the speaker seeks to secure on the part of the addressee (B\&L: 71-4). Their predictions fall short of accounting for the fact that, in speaking politely, speakers position themselves not only in relation to their addressees, but also 
in relation to the whole of the culture, with which they seek to identify themselves to a greater or lesser extent (cf. the discussion of acts of identity in Section 3 above). Consequently, the 'pay-offs' sought may transcend the dyad, and as such they may not always be possible to secure via the medium of the addressee. This is an insight which cannot be incorporated in Brown and Levinson's account, where the pay-offs sought apply exclusively at the microlevel (the level of the dyad), and such pay-offs provide the motivation for particular strategies.

An account in terms of frames which summarise speakers' choices in context, rather than trying to pre-empt them, suggests itself as a powerful tool with which to analyse polite discourse. In accounting for the CG data, we invoked speakers' desire to present themselves in a certain way, which subsumes their desire to accommodate to their addressees' speech. It emerged that, when speaking politely, speakers both are constrained by their position in social space - which also determines the varieties of speech to which they have access and use polite discourse to position themselves in it. In other words, in speaking politely, speakers also act out a social persona which transcends the narrow considerations of the dyad and transforms them into social actors who have the ability not only to passively use language, but also to actively change it. It is in this sense that we must understand Klein's observation that "where semantic systems are postulated, one must also consider the possibility of shifts in the predominant pragmatic strategies or 'norms' of actual use [...] the possibility of interaction between these two kinds of bias [...] [can] lead to the reinterpretation of pragmatic preferences as social norms" (1980:61; original emphasis). What paves the way for changes in semantic systems is speakers' active use of language: who is talking to whom, what is the relationship between them, and in what setting.

An account in terms of frames also lays a claim on psychological plausibility as an account of interlocutors' online processing of polite discourse. To the extent that variation is found in every society, and to the extent that linguistic forms are characterised as standard or vernacular, or more or less salient, based on extra-linguistic (socio-historical) and linguistic (structural) considerations, speakers cannot be operating using Brown and Levinson's formula for computing $\mathrm{W}$ and their proposed hierarchy of strategies in vacuo. The notions of $\mathrm{D}$ and $\mathrm{P}$ and of indirectness are adequate to account for what goes on synchronically only after they have been 'contextualised' taking into account the particularities of the society and of the language at hand. However, as the analyses in Section 3 show, untangling the underlying principles at work necessitates close scrutiny 
using theoretical tools a lay-person cannot be assumed to master, much less have the time to resort to during online processing.

This is where formulaicity comes in. The combinations of linguistic features analysed there provide ready-made solutions to the complex and pertinent problem of constituting one's own and one's addressee's face while simultaneously ensuring that one's immediate goals in interaction are achieved. Thus, the linguistic expressions analysed constitute "routine formulae" on both counts of Coulmas's (1994: 1292) definition: they are 'fixed' both in form, compared to other expressions in the language which are created anew every time, and in function, in that they fulfil specific highly recurrent communicative tasks.

That a large proportion - albeit by no means all — of polite discourse should consist in using formulaic speech is hardly surprising. From the point of view of online processing, psycholinguistic evidence shows that possessing a sufficiently large stock of formulae allows more time for planning thus promoting fluency in speech (Pawley \& Syder 1983; Wray \& Perkins 2000: 15-17). The normal order of acquisition by which receptive competence precedes production is also often reversed in the case of routines and politeness formulae (Saville-Troike 1989/1982:241; Wray \& Perkins 2000:19-20). Saville-Troike (ibid.) associates this reversal with the fundamental importance of using politeness formulae for effectively assuming the role of social actor. That is, formulae "embody accepted ways of responding verbally to a variety of situations [...]. Using the expected formulas is a strong indication of belonging, social identity or acculturation" (Coulmas 1994:1293; cf. Wray \& Perkins 2000:13-5). This property of formulaic speech renders it particularly apt for maintaining face, an integral part of which is demonstrating familiarity with the norms of the community within which one is operating (Terkourafi 2001b:24ff.). The psycholinguistic and interactional significance of formulaic speech may in fact be seen as two sides of the same coin:

"[T]he driving force behind the processing short-cuts is ensuring that the speaker's production is fluent and that information is available when required [...] The driving force behind the socio-interactional formulas is ensuring that the speaker gets what he/she wants and is perceived as an individual within the group. Significantly, formulaic language is better suited to this than novel language is, because a hearer is more likely to understand a message if it is in a form he/she has heard before, and which he/she can process without recourse to full analytic decoding. [...]" (Wray \& Perkins 2000: 17-8, original emphasis and spelling). 
Viewed from this perspective, our results come as no surprise: the uniformity of speakers' choices in the data collected is explained by "the speakers' selfinterest in matching their output to what the hearers will understand" (Wray \& Perkins 2000: 18 fn. 6).

The importance of formulaic speech to politeness is of course by no means a new finding. Numerous studies discuss the use of formulaic expressions to achieve specific goals in interaction (e.g., Overstreet and Yule (2001) on the

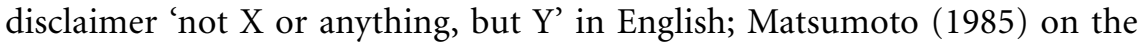
hedge 'chotto', Ide (1998) and Takekuro (1999) on the expressions 'sumimasen' and 'onegaishimasu' respectively in Japanese; Nwoye (1989) on proverbs in Igbo; Ferguson (1981) on greetings and responses to them in Arabic). What I am arguing for, however, is a much broader definition of formulaicity, and consequently its wider distribution across situational contexts. Formulaicity may be viewed as the synchronic counterpart to grammaticalisation, and can be assessed using diachronically-inspired criteria (Terkourafi 2001b: 155ff.). One significant property of formulaic expressions is arbitrariness: formulaicity cannot be predicted based on morpho-phonological, syntactic, semantic, or pragmatic criteria alone, although, jointly considered, these can provide indications of the extent to which an expression synchronically functions as formulaic. The CG results thus re-cast the standard picture both qualitatively and quantitatively: in the data collected, formulaic speech carries the burden of polite discourse. Given the psycholinguistic and interactional significance of formulaic speech, this raises the possibility that the use of formulae may be a prominent feature of polite discourse in any culture. While the confirmation of this prediction must necessarily await further quantitative studies of polite discourse across cultures, it does suggest the need for an account in which emphasis is placed, not on a universally valid principle motivating nonce inferences, but on experientially acquired structures of anticipated 'default' behaviour.

\section{Conclusion}

Rather than the application of a universally-valid principle which associates the sum of $\mathrm{D}, \mathrm{P}$ and $\mathrm{R}_{x}$ with semantically defined degrees of indirectness, the analysis of speakers' choices in context reveals a knowledge of which expressions to use in which situations. The conventional character of this knowledge is supported in three ways. First, it is constrained by such attributes as interlocutors' 
sex, age, and social class. Second, expressions which are equally indirect on semantic criteria are not used interchangeably, but are tied to observable features of the situation. The sex, age and social class of the speaker and of the addressee, the relationship between them, the setting of the exchange and the order of appearance of the speech act are extra-linguistic features of the situation available to interlocutors through perception, from which further items of background knowledge (e.g. participants' rights and obligations) are derivable. Crucially, it is the co-occurrence of particular values of these features that accounts for speakers' use of politeness formulae in the data. Joint reference to all these features is therefore necessary to delimit the range of situations to which specific formulae are applicable, though it is likely that not all will be equally determining for the use of any particular formula. Third, given a combination of extra-linguistic features, speakers' choices cluster around concrete, fully-specified linguistic realisations. In this respect, the resources of grammar and phonology are not creatively drawn upon by speakers. Rather, the consistency in speakers' choices extends to the level of phonetic detail, providing a strong indication of the formulaic character of the corresponding expressions.

\section{Notes}

1. See Figure 1. Potential realisations of strategies 2 through 4 are summarised in B\&L: 102, 131 , and 214 respectively.

2. Combinations of extra-linguistic features are, in the first instance, the conditioning factor here. Whether these can be consistently reduced to $\mathrm{W}$ values remains an open question, however; on some difficulties pertaining to this move, see Terkourafi (forthcoming).

3. These are further defined and exemplified in Section 3.

4. This is implicit in Brown and Levinson's listing under the positive, negative, and offrecord strategies potential realisations without ever discussing how one might choose amongst them. Recall that $\mathrm{W}$ values only guide the selection of a strategy, not of any particular realisation of this strategy. This move is perhaps desirable when individual languages and communities are abstracted away from, but, at the same time, limits the applicability of their proposals to one level of remove from actual discourse. By revealing contextually-conditioned preferences for particular realisations of strategies, the quantitative analysis of empirical data adds a further dimension to the situated functioning of polite discourse, one which, as large corpora of naturally-occurring discourse are becoming increasingly available and amenable to statistically-informed analyses, politeness theorists can no longer afford to ignore.

5. These features pertain to categories which are socially constructed to a greater or lesser extent. Nevertheless, they may function as input for determining $\mathrm{D}, \mathrm{P}$ and $\mathrm{R}_{x}$ values 
inasmuch as they are immediately perceivable (in the sense that, ceteris paribus, participants operate on default assumptions about them, partly based on sensory data).

6. The classification of main-clause VPs realising requests in these tables is driven by both syntactico-semantic and lexical considerations influencing indirectness. Thus, 'type of mainclause verb' encompasses not only exo, ('I-have') boro ('I-can'), and Aelo ('I-want'), lexicalising modalities such as ability and volition, but also the generic labels ACTION VERB, referring to main-clause verbs whose propositional content expresses the act to be performed when this act is not verbal (e.g. Jino, 'I-give', pjano, 'I-pass, I-take, I-buy', pino, 'I-drink'), and SPEECH ACT VERB, referring to verbs whose propositional content expresses the act to be performed when this act is verbal (e.g. milo, 'I-speak', sxoliazo, 'I-comment', apando, 'I-answer'). Further (non-lexical) devices realising modality in natural languages are captured under 'subjective modality' (Lyons 1995:328ff), a term highlighting their potential for expressing subjectivity, which makes them apt for the expression of politeness. Modality may be grammaticalised in two ways: via verbal mood (ibid.:255), and (some uses of) tense (ibid.:319). Prosodic structure may also realise modality, with rising intonation marking interrogativity in languages such as Modern Greek, where this is not signalled structurally (ibid.: 185-6; Sifianou 1992:137-9). Finally, modality may be realised by means of negation (Lyons 1995:175-6; Sifianou 1992:146ff.). As a result, in addition to grammatical mood, tense, question-intonation, and negation are also noted under 'subjective modality'.

7. In these and following frames, extra-linguistic variants are listed in order of frequency, with most frequent ones placed first.

8. Two different definitions of indirectness are proposed by Brown and Levinson; both rely on semantic criteria. According to a first definition, indirectness is "any communicative behaviour, verbal or non-verbal, that conveys something more than or different from what it literally means" (B\&L: 134). The semantic bent of this definition concerns its reliance on the notion of literal meaning, whence also the difficulties in applying it. Bach (1994) advocates a notion of literal meaning which encompasses the semantic representation of sentences together with referent assignment to deictic and referring expressions, but stops short of identifying this with the minimal truth-evaluable proposition which may be the result of further 'saturation' or 'completion'. However, theorists defending common pretheoretical intuitions as a guide to fleshing out 'what is said' by an utterance (Récanati $1989 / 1991$ ) would not be prepared to take this step, since it renders most of everyday speech non-literal, thereby going against such intuitions (for a summary of related problems, see Leezenberg 2001:300ff.).

Elsewhere (B\&L: 6-7), they adopt Leech's (1983) analysis of the offers in (i) to explain their intuitively different politeness import:

(i) a. Will you have anything to eat?

b. Will you have something to eat?

c. Won't you have anything to eat?

d. Won't you have something to eat?

Leech (1983:157ff.) proposes an analysis of these sentences as generalised conversational implicatures along Gricean lines. According to this, their semantic structure (positive vs. negative polarity of something vs. anything, non-informativeness of negative questions) is key 
to deriving the corresponding generalised implicatures. Their indirectness resides in the increasing complexity of deriving these implicatures, which is thus responsible for their increasing politeness import (cf. Leech 1980: 109ff.). Given the 'hybrid', semantico-pragmatic nature of generalised conversational implicatures (Levinson 2000), the interpretation of (a) to $(\mathrm{d})$ as increasingly polite is thus arguably, again, largely dependent on semantic input.

\section{References}

Auer, Peter, Birgit Barden \& Beate Grosskopf. 1998. Subjective and objective parameters determining 'salience' in long-term dialect accommodation. Journal of Sociolinguistics 2, 163-187.

Bach, Kent. 1994. "Semantic slack: what is said and more." Foundations of speech act theory: philosophical and linguistic perspectives, ed. by S. Tsohatzidis, 267-291. London: Routledge.

Brown, Penelope \& Stephen Levinson. 1987/1978. Politeness: some universals in language usage. Cambridge: Cambridge University Press. Reprinted from: Questions and politeness: strategies in social interaction, ed. by E. Goody, 56-234. Cambridge: Cambridge University Press.

Coulmas, Florian. 1994. "Formulaic language.” Encyclopedia of Language and Linguistics, ed. by N. Asher, Vol. 3. 1292-3.

Ferguson, Charles. 1981. "The structure and use of politeness formulas." Conversational routine: explorations in standardised communication situations and prepatterned speech, ed. by F. Coulmas, 21-35. The Hague: Mouton.

Geis, Michael. 1995. Speech acts and conversational interaction. Cambridge: Cambridge University Press.

Ide, Sachiko. 1998. “'Sorry for your kindness': Japanese interactional ritual in public discourse." Journal of Pragmatics 29, 509-529.

Klein, Flora. 1980. "Pragmatic and sociolinguistic bias in semantic change." Papers from the 4th international conference on historical linguistics, ed. by E. Closs Traugott, R. Labrum \& S. Shepherd, 61-74. Amsterdam: John Benjamins.

Leech, Geoffrey. 1980 "Language and tact." Explorations in semantics and pragmatics. Pragmatics and Beyond 4. 79-117. Amsterdam: John Benjamins.

Leech, Geoffrey. 1983. Principles of pragmatics. London: Longman.

Leezenberg, Michiel. 2001. Contexts of metaphor. Oxford: Elsevier Science.

Le Page, Robert \& Tabouret-Keller, Andrée. 1985. Acts of identity: creole-based approaches to language and ethnicity. Cambridge: Cambridge University Press.

Levinson, Stephen C. 2000. Presumptive meanings: the theory of generalised conversational implicature. Cambridge, MA: MIT Press.

Lyons, John. 1995. Linguistic semantics. Cambridge: Cambridge University Press.

Matsumoto, Yoshiko. 1985. “A sort of speech act qualification in Japanese: chotto." Journal of Asian Culture 9, 143-159.

Newton, Brian. 1972. Cypriot Greek: its phonology and inflections. The Hague: Mouton.

Nwoye, Onuigbo. 1989. “Linguistic politeness in Igbo.” Multilingua 8, 259-275. 
Overstreet, Maryann \& George Yule. 2001. “Formulaic disclaimers.” Journal of Pragmatics 33, 45-60.

Pawley, Andrew \& Frances Syder. 1983. "Two puzzles for linguistic theory: nativelike selection and nativelike fluency." Language and communication, ed. by J.C. Richards \& R.W. Schmidt, 191-226. London: Longman.

Récanati, François. 1989/1991. “The pragmatics of what is said.” Pragmatics: a reader, ed. by S. Davis, 97-120. Oxford: Oxford University Press. 97-120. Reprinted from: Mind and Language 4, 295-329.

Saville-Troike, Muriel.1989/1982. The ethnography of communication: an introduction. Oxford: Blackwell. Second edition.

Sifianou, Maria. 1992. Politeness phenomena in England and Greece: a cross-cultural perspective. Oxford: Clarendon Press.

Sperber, Dan \& Deirdre Wilson. 1995/1986. Relevance: communication and cognition. Oxford: Blackwell. Second edition.

Takekuro, Makiko. 1999. "Formulaic speech and social conventions in linguistic politeness of Japanese." Paper presented at the International Symposium for Linguistic Politeness, Bangkok, 7-9 December 1999.

Terkourafi, Marina. 2001a. "The use of the plural in expressing politeness in Cypriot Greek conversations." Proceedings of the 4th International Conference on Greek Linguistics, Lefkosia 17-19 September 1999, 462-470.

Terkourafi, Marina. 2001b. Politeness in Cypriot Greek: a frame-based approach. Ph.D. dissertation. University of Cambridge.

Terkourafi, Marina. forthcoming. Testing Brown and Levinson's (1987) three sociological variables with reference to a corpus of spontaneous conversational data from Cypriot Greek: evidence from address terms and realisations of offers and requests. International Journal of the Sociology of Language.

Wray, Alison \& Michael Perkins. 2000. "The functions of formulaic language: an integrated model.” Language and Communication 20, 1-28.

\section{$\Pi \varepsilon \rho i ́ \lambda \eta \psi \eta$}

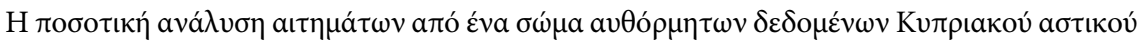

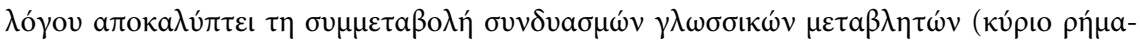

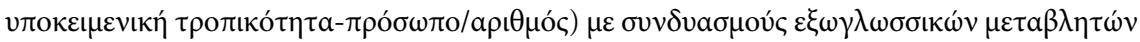

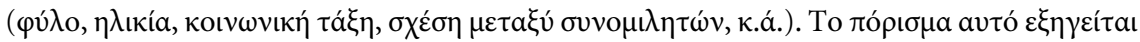

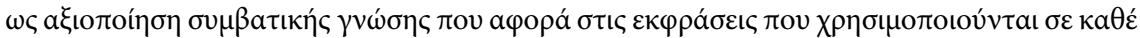

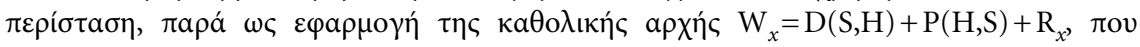

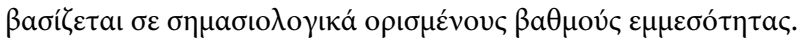

